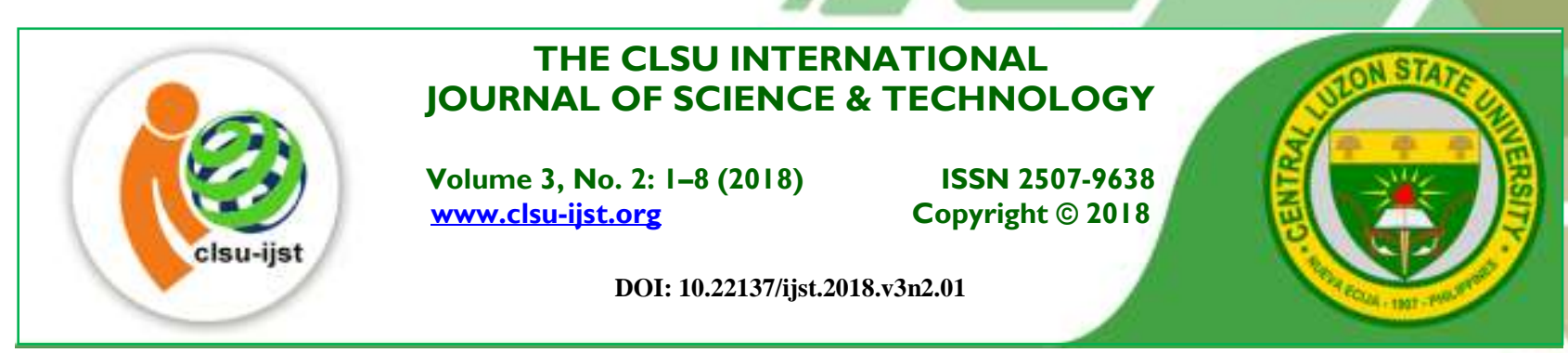

\title{
Suitability of Danish Bilharziasis Laboratory Technique (DBL) as Detection Test for Trematode Infection in Buffaloes
}

\author{
John Philip Lou M. Lumain ${ }^{1}$ and Lotis M. Balala ${ }^{1}$
}

${ }^{1}$ College of Veterinary Medicine, Visayas State University, Visca, Baybay City, Leyte, Philippines

Email for correspondence: johnphiliploum.lumain@gmail.com

\section{ABSTRACT}

Trematodes are one of the devastating parasites in ruminants with significant public health and economic importance. Continued evaluation and development of novel detection methods for accurate diagnosis are highly warranted. This study aimed to assess the suitability of the Danish Bilharziasis Laboratory (DBL) technique as a detection test for Fasciola spp. and Paramphistomum spp. infection in buffaloes. Sensitivity and specificity were compared to post-mortem recovery of parasites from specific organs. A total of 246 buffaloes were examined in this study, of which 125 (50.81\%) were infected with Fasciola spp. and 109 (44.31\%) with Paramphistomum spp. by post-mortem detection. In contrast, 107 (43.50\%) were positive for Fasciola spp. and $92(37.40 \%)$ for Paramphistomum spp. by the DBL method. Sensitivity of DBL was high in detecting Fasciola spp. (85.6\%) and Paramphistomum spp. (84.4\%). The specificity was $100 \%$ for both parasites. Kappa coefficients (к) indicated an almost perfect agreement of the two tests in detecting Fasciola spp. (0.8539) and Paramphistomum spp. (0.8577). Based on these findings, DBL is a suitable technique for the detection of trematodes in buffaloes.

Keywords: DBL, Fasciola, Paramphistomum, sensitivity, specificity

\section{INTRODUCTION}

Foodborne trematodiasis is one of the neglected tropical diseases affecting primarily the poor and marginalized people in rural settings of developing countries (Mas-coma et al., 2009). This disease is caused by trematodes which include infectious flukes such as Fasciola spp. and Paramphistomum spp. that are endemic worldwide. The flukes are two of the most common trematodes affecting ruminants resulting to vast economic loss in meat and milk production. These parasites also exhibit broad zoonotic reservoir with cattle and buffaloes being the most important natural end hosts.

Fasciola spp. which induces fascioliasis is responsible for the inflammation of the bile duct, formation of gallstone, and fibrosis of the liver in infected animal. Paramphistomum spp., on the other hand, produces severe economic loss to milk production as the fluke saps nutrients from their hosts, resulting to weight loss and decrease in milk production (Haridy et al., 2002). Because of their economic impact, development of a highly sensitive and specific diagnostic tool is warranted to achieve successful prevention and control of parasitic infection. Direct coprological diagnostics, immunodiagnostics, molecular diagnostics, and imaging techniques are the main ante-mortem tools to 
diagnose trematode infections (Keiser et al., 2006). Among these, coprological exam has advantage of being cost-effective, relatively easy to perform, and is most practical in resource-constrained settings (Pullan and Brooker et al., 2008). Several coprodiagnostic methods have been used to recover fluke eggs including flotation, Kato- Katz thick smear, and the formalin-ether/ethyl acetate concentration techniques (WHO, 2009). However, none of these techniques are satisfactory (Sindberg et al., 2014). The Danish Bilharziasis Laboratory technique (DBL), developed at DBL- Centre for Health Research and Development, is a novel fecalysis technique combining filtration and centrifugation (Willingham et al., 1998). The DBL method is gaining popularity in detecting eggs of small trematodes in domestic animals. It has been found suitable in quantifying eggs of fish-borne zoonotic trematodes in dogs, cats, and pigs (Anh et al., 2008; Sindberg et al., 2014). However, the use of DBL in detecting trematodes in buffaloes has not been explored. We therefore investigated the suitability of the DBL technique in detecting the eggs of Fasciola spp. and Paramphistomum spp. in buffaloes and compared sensitivity and specificity to the post-mortem detection of flukes in specific organs being the gold standard.

\section{MATERIALS AND METHODS}

Fecal collection in buffaloes. The study involved 246 randomly selected buffaloes at the Ormoc City Abattoir, Ormoc City, Leyte, Philippines. The sample size was determined using the formula of Cannon and Roe (1982) based on the 20\% prevalence reported by Bantugan (2004, unpublished). Prior to slaughter, 100 grams of feces were collected directly from the rectum using a lubricated hand glove. The glove later served as temporary container of the fecal material upon removal from the hand. Fecal samples were properly labeled and stored at $4^{\circ} \mathrm{C}$ until use.

Fecal examination for eggs of trematodes. The DBL technique described by Willingham et al. (1998) was used in screening fecal samples for the presence of trematode eggs. Briefly, 35 grams of fecal sample were homogenized in a mortar and pestle to allow even distribution of the eggs in the feces. After homogenization, five grams of subsamples were washed in saline solution $(1.2 \%)$ and shaken mechanically for 10 minutes in a shaker machine. The fecal mixture was then poured into a series of sieve stacks measuring $400 \mu \mathrm{m}, 100 \mu \mathrm{m}$, and $45 \mu \mathrm{m}$ from top to bottom. The residual sediments were washed with saline and allowed to settle for 10 minutes. Washing with saline solution was repeated with in between discharge of the supernatant. Retained sediments were centrifuged at $1500 \mathrm{rpm}$ for 3 minutes and resuspended in saline to $2.25 \mathrm{~mL}$ volume. A drop of methylene blue was then added in the preparation to provide a good contrast for the visualization of eggs (Hansen \& Perry, 1994). About $150 \mu \mathrm{l}$ of the fecal suspension was mixed with $850 \mu \mathrm{l}$ of saline solution in a DBL slide for microscopic examination. The presence of the eggs of Fasciola spp. and Paramphistomum spp. in the feces was recorded "positive" and "negative" if eggs were not detected.

Trematode egg identification. The eggs were identified based on the description provided by Soulsby (1982). Eggs of Fasciola spp. were operculated, ovoid in shape, light yellow to greyish in color with length ranging from 130 to $150 \mu \mathrm{m}$ and width from $60-90 \mu \mathrm{m}$. The eggs of Paramphistomum spp. were operculated, ovoid in shape, brown to greenish in color with a length ranging from 114-176 $\mu \mathrm{m}$ and width from 73-100 $\mu \mathrm{m}$. The eggs were further distinguished through the diffusion of methylene blue in the eggs of Paramphistomum (Figure 1) 35-40 minutes after staining due to thinner egg capsule "(Hansen \& Perry, 1994). The eggs were viewed at 400x magnification and measured by ocular micrometry with $26 \mu \mathrm{m}$ per ocular division (Zajac, 2012). 

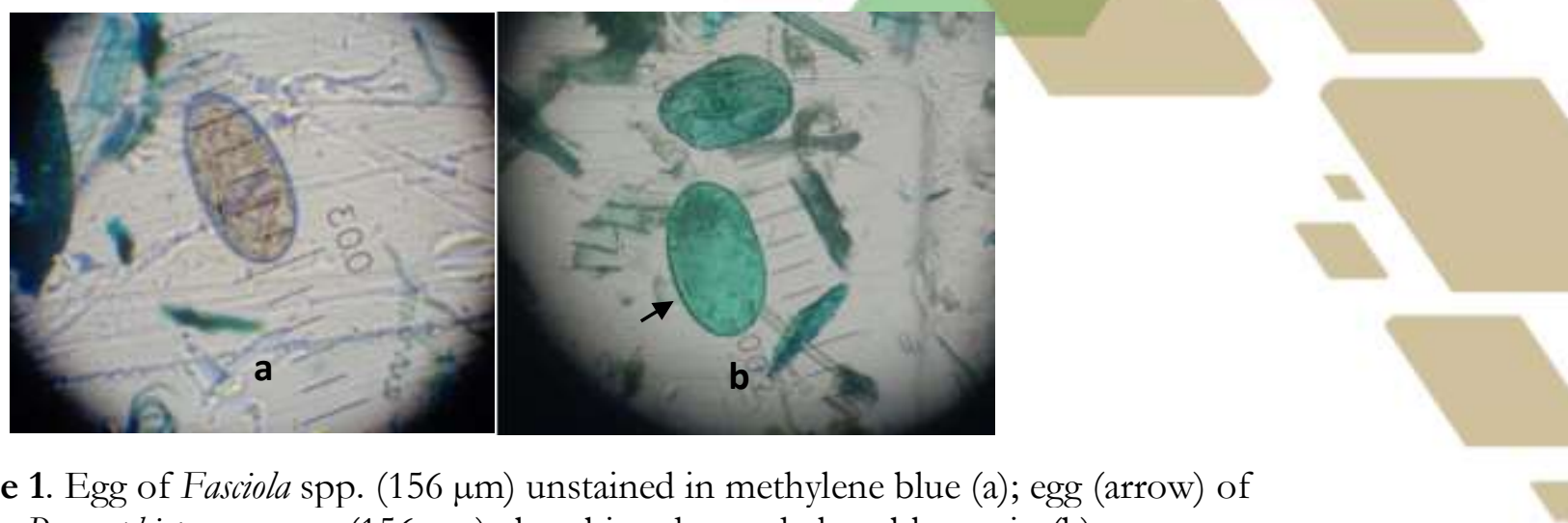

Figure 1. Egg of Fasciola spp. $(156 \mu \mathrm{m})$ unstained in methylene blue (a); egg (arrow) of Paramphistomum spp. $(156 \mu \mathrm{m})$ absorbing the methylene blue stain (b) at 400x magnification.

Post-mortem detection of parasites. Post-mortem recovery of trematodes in their predilection sites was the gold standard in concluding positive infection in the animals. After slaughter, the liver and forestomach of each of the sampled animal were removed for visual inspection. To detect Fasciola spp., the common bile duct and the gall bladder were palpated and cut-open using a scalpel blade. Paramphistomum spp. was traced from the rumen, reticulum, omasum, and abomasum. The stomach contents were flushed with running water to visualize parasites adhering to the stomach wall. The recovery of trematodes was regarded as "positive" and "negative" if undetected.

Adult parasite identification. Species of Fasciola and Paramphistomum were identified based on morphological characteristics described by Soulsby (1982). Briefly, Fasciola hepatica is leaf-shaped with broad shoulders and may reach a size of $30 \mathrm{~mm}$ in length and $13 \mathrm{~mm}$ in breadth. Fasciola gigantica, though resembling Fasciola hepatica, is recognized by its larger size that is $25-75 \mathrm{~mm}$ in length and up to $12 \mathrm{~mm}$ in breadth, shoulders are not prominent, and the body is more transparent. Paramphistomum is pear-shaped, slightly concave ventrally and converse dorsally, with a large posterior subterminal sucker. It measures $5-13 \mathrm{~mm}$ in length, and $2-5 \mathrm{~mm}$ in breadth. It is light red in color when alive.

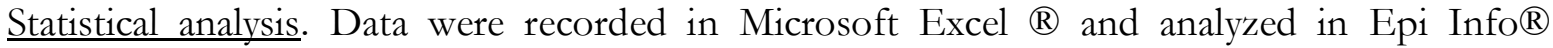
version 7. Prevalence was computed based on the formula:

\section{Prevalene Proportion $=\frac{\text { Number of infected uninul }}{\text { Tolal sumber of tonimal examined }} \times 100$}

A Chi-square test was used to compare the frequency of parasitic infections and considered significant at $p<0.05$. Diagnostic sensitivity was calculated as the probability that a true positive animal was classified as positive while diagnostic specificity was calculated as the probability that a true negative animal was classified negative (Anh et al., 2008). Kappa coefficients ( $\mathrm{\kappa}$ ) was interpreted using the guidelines outlined by Landis and Koch (1977), where strength of the kappa coefficients is slight (0.01-0.20), fair (0.21-0.40), moderate $(0.41-0.60)$, substantial $(0.61-0.80)$, and almost perfect $(0.81$ $1.00)$. 


\section{RESULTS AND DISCUSSION}

In this study, 256 buffaloes were examined using DBL technique and post-mortem recovery of parasites from their predilection sites. The latter was used as gold standard to evaluate diagnostic sensitivity and diagnostic specificity of DBL. Originally, DBL was developed to detect Schistosoma japonicum in animals by the Danish Centre for Health Research and Development (Willingham et al., 1998). It was later used to screen buffaloes for S. japonicum in Western Samar and Sorsogon Provinces in the Philippines (Carabin et al., 2005). Table 1 outlines the prevalence of trematodes in buffaloes using the two methods. The prevalences of Fasciola spp. and Paramphistomum spp. were higher by postmortem detection of parasites $(\phi<0.05)$. There was generally a higher prevalence of Fasciola spp. compared to Paramphistomum spp. Co-infection was significantly higher by post-mortem detection compared to DBL $(p<0.05)$. The occurrence of co-infection is parallel in many studies where gastrointestinal nematodes are also present aside from the trematodes. Fasciola spp. and Paramphistomum spp. are among the most prevalent gastrointestinal helminths in ruminants (Raza, et al., 2007; Mamun, et al., 2011). Most of the buffaloes examined in this study were owned by smallhold farmers who have limited resources to maintain good management and improve the health status of the animals. More often, deworming was not applied due to financial constraint.

Table 1. Prevalence of Fasciola spp. and Paramphistomum spp. in buffaloes

\begin{tabular}{lcccc}
\hline & \multicolumn{2}{c}{ Post-mortem detection } & \multicolumn{2}{c}{ DBL technique } \\
\cline { 2 - 5 } & $\begin{array}{c}\text { Positive } \\
(\mathbf{n}=\mathbf{2 5 6})\end{array}$ & Prevalence (95\% CI) & $\begin{array}{l}\text { Positive } \\
(\mathbf{n = 2 5 6 )}\end{array}$ & Prevalence (95\% CI) \\
\hline Fasciola spp. & 125 & $50.81 \pm 0.09$ & 107 & $43.50 \pm 0.09$ \\
Paramphistomum spp. & 109 & $44.31 \pm 0.09$ & 92 & $37.40 \pm 0.10$ \\
Co-infection & 79 & $32.11 \pm 0.10$ & 66 & $26.83 \pm 0.11$ \\
\hline
\end{tabular}

The presence of helminths in ruminants can be predisposed by some factors such as host age, sex and breeding status, and grazing habits. Additional factors are the level of education and economic capacity of farmers and their standard of management (Asanji \&Williams, 1987; Gulland \& Fox, 1992). In addition, geoclimatic conditions and poor awareness of livestock farmers contribute towards a conducive environment for the development and growth of parasites (Raza et al., 2007). The prevalence of Fasciola gigantica in this study was similar to the report of Molina et al. (2010), while prevalence of Paramphistomum cervi is higher than in the study of Iqbal et al. (2013). Dorny et al. (2011) reported prevalence estimates of 5-20\% and 45-95\% for Fasciola and Paramphistomum, respectively, using coprological examination. Lower prevalence (Fasciola hepatica - 32\% and Paramphistomum cervi 11\%) was reported by Kobak and Pilarczyk (2012) in water buffaloes raised in the Notecka Forest Region in Poland. This regional variation may be attributed to different geographical distributions, host factors, and climatic conditions required for the development of free-living stages of the trematodes (Raza et al., 2007). Throughout South East Asia, infections with gastrointestinal trematodes are very common in ruminants because of appropriate climatic conditions year-round that favor the development and transmission of the infective stages of trematodes from grasslands (Iqbal et al., 2013; Dorny et al., 2011). Buffaloes that are usually kept tied in a stick in large natural pasture may acquire the infective metacercaria (Faylon, 1992). In addition, the propensity of buffaloes to seek rivers, pools, or swamps for wallowing raises the risk of infection to snail-borne helminthes (Cockrill, 1974).

\section{Vol. 3 No. 2 ISSN: 2507-9638 DOI: 10.22137/ijst.2018.v3n2.01}

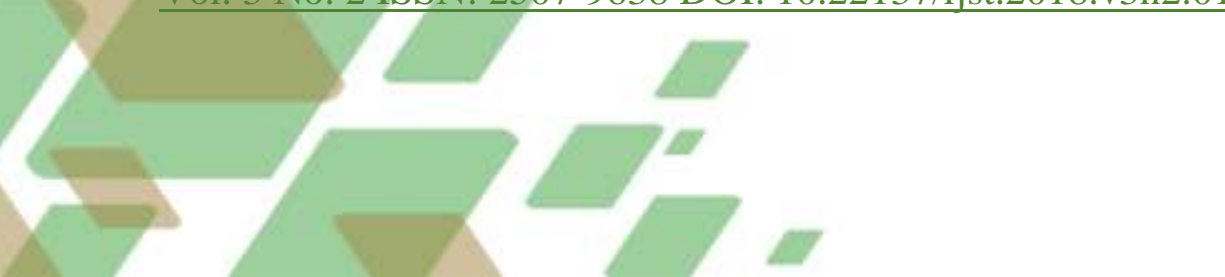


Table 2. Specificity and sensitivity of DBL as detection test for trematodes

\begin{tabular}{lccccc}
\hline & $\begin{array}{c}\text { Post-mortem } \\
\text { detection }\end{array}$ & DBL & $\begin{array}{c}\text { Sensitivity } \\
\mathbf{( \% )}\end{array}$ & $\begin{array}{c}\text { Specificity } \\
\mathbf{( \% )}\end{array}$ & $\begin{array}{c}\text { Kappa } \\
\text { coefficients }\end{array}$ \\
\hline Fasciola spp. & 125 & 107 & 85.6 & 100 & 0.8539 \\
Paramphistomum spp. & 109 & 92 & 84.4 & 100 & 0.8577 \\
Co-infection & 79 & 66 & 83.5 & 100 & 0.8733 \\
\hline
\end{tabular}

Table 2 shows that the sensitivity of DBL to detect Fasciola spp. and Paramphistomum spp. was $85.6 \%$ and $84.4 \%$, respectively. Specificity was $100 \%$ for both parasites. So far, no data is available to compare sensitivity and specificity of DBL in detecting trematodes in buffaloes. However, Sindberg et al. (2013) recorded an 85\% sensitivity of DBL in detecting small trematode eggs in dog feces. The same level of sensitivity (80-92\%) was also reported by Anh et al. (2008) for small trematodes, mostly Heterophyidae and Echinostomatidae belonging to the genera Haplorchis and Echinochasmus, in dogs, cats, and pigs. Specificity was $100 \%$ in all animal species. In this study, the specificity of DBL was high, however, a $100 \%$ specificity may be unreal if the possibility of false positives data is to be considered (Tarafder et al., 2010). It should also be noted that test sensitivities are strongly influenced by intensity of infection and this variation needs to be taken into account for the choice of a diagnostic test in a specific setting (Nikolay et al., 2014). Furthermore, the kappa coefficients ( $\kappa$ ) indicated an almost perfect agreement between DBL and post-mortem recovery in detecting Fasciola spp. (0.8539) and Paramphistomum spp. (0.8577) with $92.68 \%$ and $93.08 \%$ degree of agreement, respectively. This confirms the suitability of DBL as detection test for trematodes in buffaloes.

Results of this study show that DBL can be a satisfactory detection test in the field or any setting where slaughter or necropsy of the buffaloes is not necessitated. As a test, DBL offers several advantages over other detection methods being simple, quantitative, and does not require special reagents or equipment to perform the examination (Ahn et al., 2008). This technique can easily be performed by even the simplest veterinary laboratory to produce data that are particularly important for epidemiological analyses.

\section{CONCLUSION}

Occurrence of trematodes continues to affect development in the buffalo industry. The deleterious effect of these parasites led to production losses and condemnation of affected organs after slaughter. A highly sensitive and specific diagnostic tool is therefore appropriate to design effective control strategies. To the best of our knowledge, the present study is the first to publish data on the sensitivity and specificity of DBL in detecting trematodes in buffaloes. The results highly suggest considering DBL as a tool for routine diagnosis. Moreover, the use of serological and molecular tools may be considered to address concerns in sample processing, low infection rate, and overlapping morphological features.

\section{Acknowledgments}

The authors are grateful to Drs. Eugene B. Lañada, Naomi Ruth Q. Torregoza, and Froiland E. Sanchez for their support and expert assistance extended to this study. Likewise, to the personnel of the Diagnostic Laboratory of the College of Veterinary Medicine, Visayas State University, Visca, Baybay City and Ormoc City Abattoir, Ormoc City.

\section{Vol. 3 No. 2 ISSN: $2507-9638$ DOI: $10.22137 /$ ijst.2018.v3n2.01}




\section{REFERENCES}

Anh, N.T.L., Phuong, N.T., Thu, L.T., Johansen, M.V., Murrell, D.K. and Thamsborg, S.M., 2008. Evaluation of techniques for the detection of small trematode eggs in feces of domestic animals. Veterinary Parasitology. 156: 346-349.

Asanji, M.F. and Williams, M.O. 1987. Variables affecting population dynamics of gastrointestinal helminth parasites of small farm ruminants in Sierra Leone. Bulletin of Animal Health and Production, Africa 35: 308-113.

Bantugan, S.E. 2004. Trematode Infections Among Carabaos (Bubalus bubalis carabenensis) in Mayorga Leyte; Prevalence and association risk factors. Undergraduate Thesis. College of Veterinary Medicine. Leyte State University, Visca, Baybay, Leyte. Philippines

Canon, R.M. and Roe, R.T. 1982. Livestock disease surveys: a field manual for veterinarians. Australian Government Publishing Service, Canberra, Australia.

Carabin, H., Balolong, E., Joseph, L., McGarvey, S.T., Johansen, M.V., Fernandez, T., Willingham, A.L. and Olveda, R. 2005. Estimating sensitivity and specificity of a fecal examination method for Schistosoma japonicum infection in cats, dogs, water buffaloes, pigs, rats in Western Samar and Sorsogon provinces, Philippines. International Journal of Parasitology. 35: 1517-1524.

Cockrill, W.R. 1974. The working buffalo. In: Husbandry and Health of the Domestic Buffalo. Food and Agriculture Organization of the United Nations, Rome, Italy.

Dornya, P.B., V. Stoliaroffa, J. Charliera, S. Measc, S. Sornd, B. Cheac, D. Holld, D.V. Akene and Vercruyssea, J. 2011. Infections with gastrointestinal nematodes, Fasciola and Paramphistomum in cattle in Cambodia and their association with morbidity parameters. Veterinary Parasitology. 175: 293-299.

Faylon, P.S. 1992. Buffaloes Development in the Philippines. In: Buffaloes Production in the Philippines. Edited by S.K. Ranjhanand P.S Faylon. Philippine Council for Agricultural, Forestry and Natural Resource Development, Los Baňos, Laguna.

Gulland, F.M.D. and Fox, M. 1992. Epidemiology of nematode infections of Soy sheep (Ovies aries L.) on St. Kilda. Parasitology. 105: 481-492.

Hansen, J. and Perry, B. 1994. The epidemiology, diagnosis and control of helminth parasites of ruminants. A handbook, International laboratory for research on Animal Disease. Nairobi, Kenya. pp. 31-36.

Haridy, F. M., T. A. Morsy, N. I. Gawish, T. N. Antonios and Gawad, A., 2002. The potential reservoir role of donkeys and horses in zoonotic fascioliasis in Gharbia Governorate, Egypt. Journal of the Egyptian Society of Parasitology. 32(2): 561-570.

Igbal, M.N., K.A. Shahzadb and Muhammad, A. 2013. Identification and prevalence of Paramphistomum cervi in naturally infected water buffaloes of Central Punjab, Pakistan. Veterinaria. 1(1): 9-12.

\section{Vol. 3 No. 2 ISSN: 2507-9638 DOI: 10.22137/ijst.2018.v3n2.01}




\section{-}

Johansen, M.V., Sithithaworn, P., Bergquist, R., and Utzinger, J. 2010. Towards improved diagnosis of zoonotic trematode infections in Southeast Asia. In: Rollinson, D., Hay, S.I. (Eds.) Advances in parasitology, Vol 73. Academic Press: Burlington. pp. 171-195.

Keiser, J., Utzinger, J., Tanner, M., Dong, Y., and Vennerstorm, J.L. 2006. The synthetic peroxide OZ78 is effective against Echinostoma capronia and Fasciola hepatica. Journal of Antimicrobial Chemotherapy. 58(60): 1193-1197.

Kobak, P. and Pilarczyki, B. 2012. Prevalence of gastrointestinal parasites of water buffaloes raised in the Notecka Forest Region (Poland). Bulletin of the Veterinary Institute in Pulawy. 56: 33-36.

Landis, J.R. and Koch, GG. 1977. The measurement of observer agreement for categorical data. Biometrics.33(1):159-174.

Mamun, M.A.A., N. Begum and Mondal, M.M.H. 2011. A coprological survey of gastro-intestinal parasites of water buffaloes (Bubalus bubalis) in Kurigram district of Bangladesh. Journal of Bangladesh Agricultural University. 9(1): 103-109.

Mas-coma, S. and Bargues, M.D. 1997. Human liver flukes: a review. Research and Reviews in Parasitology. 57:145-218.

Mas-coma S., Bargues, M. D., and Valero, M.A. 2005. Fascioliasis and other plant-borne trematode zoonoses. International Journal of Parasitology. 35 (11-12): 1255-1278.

Mas-Coma S, Valero MA, and Bargues MD. 2009. Chapter 2: Fasciola, lymnaeids and human fascioliasis, with a global overview on disease transmission, epidemiology, evolutionary genetics, molecular epidemiology and control. Advances in Parasitology. 69: 41-149.

Molina, E.C., Navarra, G.A., and Aloot, P.O. 2010. Fasciolosis in cattle and carabaos in selected barangays of Pikit and Kabacan, Cotabato, Philippines. USM R and D. 18(2): 129-132.

Parikh, R., Mathai, A., Parikh, S., Sekhar, G. C., and Thomas, R. 2008. Understanding and using sensitivity, specificity and predictive values. Indian Journal of Ophthalmology. 56 (1): 45-50.

Pullan, R. and Brooker, S. 2008. Sensitivity of diagnostic tests for human soil-transmitted helminth infections: a meta-analysis in the absence of a true gold standard. International Journal of Parasitology. 44: 765-774.

Raza, M.A., Iqbal, Z., Jabarrl, A., and Yaseen M. 2007. Point prevalence of gastrointestinal helminthiasis in ruminants in Southern Punjab, Pakistan. Journal of Helminthology. 81: 323-328.

Sindberg, D., Nissen, S., Anh, N.T.L., and Johansen, M.V. 2013. Evaluation of four coproscopic techniques for detection of small trematode eggs in dogs feces. International Journal of Parasitology. 195: 192-197.

Soulsby, E.J.L. 1982. Helminths, arthropods and protozoa of domestic animals. $6^{\text {th }}$ Edition. Balliere, Tindall and Cabell, London, England: UK 
Spithill, T.W., Smooker, P.M., and Copeman, B. 1999. Fasciola gigantica: epidemiology, control, immunology and molecular biology. In: Fasciolosis. (Dalton J.P. ed) CABI publishing, Wallingford, UK: $1-30$.

Steinmann, P., Keiser, J., Bos, R., Tanner, M., and Utzinger, J. 2006. Schistosomiasis and water resources development: systemic review, meta-analysis, and estimates of people at risk. Lancet Infectious Diseases. 6(7): 411-425.

Tarafder, M.R., Carabin, H., Joseph, L., Balolong, E. Jr., Olveda, R., and McGarvey, S.T. 2010. Estimating the sensitivity and specificity of Kato-Katz stool examination technique for the detection of hookworms, Ascaris lumbricoides and Trichuris trichiura infections in humans in the absence of a gold standard. International Journal of Parasitology. 40: 399-404.

Valero, M.A., Darce, N.A., Panova, M., and Mas-coma, S. 2001. Relationships between host species and morphometric patterns in Fasciola hepatica adults and eggs from the Northern Bolivian Altiplano hyperendemic region. Veterinary Parasitology. 102:85-100.

WHO. 2009. Position paper on foodborne trematode (FBT) infections. WHO Expert Meeting on Foodborne Trematode Infections and Taeniasis/Cysticercosis in Vientiane, Lao People's Democratic Republic, 12-16 October 2009. World Health Organization, Geneva.

Willingham, A.L., Johansen, M.V., and Barnes, E.H. 1998. A new technic for counting Schistosoma japonicum eggs in pig feces. Southeast Asian Journal of Tropical Medicine and Public Health. 29: 128130.

Zajac, A.M. and Conboy, G.A. 2012. Veterinary Clinical Parasitology, 8th edn. A John Wiley \& Sons, Inc., Publication, UK. 